\title{
Microbial synthesis of human-hormone melatonin at gram scales
}

Hao Luo, ${ }^{*}, 1$ Konstantin Schneider, ${ }^{1}$ Ulla Christensen, ${ }^{1}$ Lei Yang, ${ }^{1}$ Markus Herrgard, ${ }^{1}$

Bernhard Ø. Palsson ${ }^{1,2,3}$

${ }^{1}$ Novo Nordisk Foundation Center for Biosustainability, Technical University of Denmark, Kongens Lyngby 2800, Denmark

${ }^{2}$ Department of Bioengineering, University of California, San Diego, La Jolla, California 92093, United States of America

${ }^{3}$ Department of Pediatrics, University of California, San Diego, La Jolla, California 92093, United States of America

Supplementary table

\begin{tabular}{|c|c|c|c|}
\hline \multicolumn{4}{|c|}{$D d c$} \\
\hline Species & $\begin{array}{l}\text { NCBI or Uniprot } \\
\text { Accession number }\end{array}$ & $\begin{array}{c}\text { Relative activity on } \\
\text { TRP }\end{array}$ & $\begin{array}{l}\text { Relative activity on } \\
\text { 5HTP }\end{array}$ \\
\hline $\begin{array}{l}\text { Candidatus Koribacter versatilis } \\
\text { Ellin345 }\end{array}$ & ABF41161.1 & + & ++++ \\
\hline Draconibacterium orientale & AHW60462.1 & - & ++ \\
\hline Homo sapiens & P20711-1 & - & ++ \\
\hline Nisaea denitrificans & WP_028467075.1 & - & ++ \\
\hline Ophiorrhiza pumila & BAC41515.1 & - & + \\
\hline Oryza sativa Japonica Group & NP_001060968.1 & ++++ & ++++ \\
\hline Streptomyces griseofuscus & AHL44346.1 & ++ & ++ \\
\hline \multicolumn{4}{|c|}{ Aanat } \\
\hline Species & $\begin{array}{l}\text { NCBI or Uniprot } \\
\text { Accession number }\end{array}$ & \multicolumn{2}{|c|}{ Relative activity on $5 \mathrm{HT}$} \\
\hline Bos taurus & DAA18183.1 & \multicolumn{2}{|c|}{++} \\
\hline
\end{tabular}




\begin{tabular}{|c|c|c|}
\hline Gallus gallus & NP_990489.1 & +++ \\
\hline Oryctolagus cuniculus & XP_008249128.1 & +++ \\
\hline Homo sapiens & NP_001079.1 & ++++ \\
\hline Streptomyces griseofuscus & AHL44344.1 & ++++ \\
\hline \multicolumn{3}{|c|}{ Asmt } \\
\hline Species & $\begin{array}{l}\text { NCBI or Uniprot } \\
\text { Accession number }\end{array}$ & Relative activity on AcHT \\
\hline Bos taurus & P10950-1 & ++ \\
\hline Gallus gallus & NP_990674.1 & - \\
\hline Rattus norvegicus & NP_653360.2 & - \\
\hline Homo sapiens & P46597-1 & ++++ \\
\hline Elephantulus edwardii & XP_006902482.1 & + \\
\hline Takifugu rubripes & XP_011609423.1 & +++ \\
\hline Macaca mulatta & NP_001028112.1 & +++ \\
\hline \multicolumn{3}{|c|}{$\begin{array}{l}\text {-: no activity such that no desired product was formed in a biotransformation assay using whole cells } \\
+: \text { some activity such that } 20 \% \text { or fewer fed substrates were converted into the desired product in a } \\
\text { biotransformation assay using whole cells } \\
++++ \text { strong activity such that } 60 \% \text { or more fed substrates were converted into the desired product in a } \\
\text { biotransformation assay using whole cells }\end{array}$} \\
\hline
\end{tabular}


Supplementary DNA sequences 
pHM4

ccctgttatccctaggaagaaagcgaaaggagcgggcgetagggcgetggcaagtgtagcggtcacgetgegcgtaagtgcatcatccggcacttaggtatcttt caggatctcgatgctctgggtatacggattgaaataaacgctaaacggacgggtaatgcttttggcaaaatcgcgcatttttctttggcctcttcaaagctttcgctaac gaaataggcttcttgaaaggtggtaatcagacattcttgcagacaggtggttttcggatcaaatgctttaacacatgctttatcgctcagtgcatgtttcagttcaccaat gctgctcagcagacctgcaccatatgcacgcagctgaccctcttgtttgcacaggccaaattcaatggtaaagaaataacaggttgccagtttctgcacatcttcatca cttgcacccaggcttgccagaccaatttcttggctaaactgtgcaaatttcggatctgccagcagcggcacatgacccagcagttcatgacaggtatccggttccgg agtatacagcggatcgctaccatggcgaatatactgggtacaatgaaaaacacgatatgccagacctgccagaaaatcacgcggactcagataacctgcaaccg gacgaacggtaaaaccgctacgttctttcagaaacatgctcacatcttccagctgcggaacgttatcttcacgataaccacaatatttggtcagcagcggaaagttttt cagatattcacgacatgcatgggtcggatacagtttgctcagttcacgaaaaacaacaccccaggtttggtttcttcttcggtatattcaacacgcggaatcggctga ccatatttgtaacccattgcaacatcaacgaaatatttgcgacgetgacgataaacattatctttaaaacceggatgatctgcatccagttcactaccatacatcagaac acgatggctacatttatccagttcgctaattttacgcggaaaccacggaacatcttccagttccattttactcetgtgtgaaattgttatccgctcacaattccacacatta tacgagccggatgattaattgtcaaccaacattgcgaccgacggtccetcttcgctattacgccagctggcgaaagggggatgtgctgcaaggcgattaagttggg taacgccagggttttcccagtcacgacgttgtaaaacgacggccagtgagcgcgacgtaatacgactcactatagggcgaattgaaggaaggccgtcaaggccg catagggagtgtaaatttatctatacagaggtaagggttgaaagcgcgactaaattgcctgtgtaaataaaaatgtacgaaatatggattgaaaactttactttatgtgtt atcgttacgtccaacagaacatattgactatccggtattacceggcatgacaggagtaaaaatgaatctgctggaactgagctgtacaccgcagcatggtcagagtc cgctggcagataataccgtgaaacagctgctgagcaccetgccggattgggaaatcgttggtattgaactgcgtaaaacctatcgtttcgccaactatcatgaaacc atggcatttgttaatgcactggcatggattgccaatcaagaagatcatcatccggatatgtccgtgcattataatcgtgcagttgtgaacttcagcacccatgatgcag gtggtctgaccetgaatgacttcatctgtgcagcgaaaaccgaagcactgtttcgtcgtccgtaagtgccggatgattcacatcaccggcacccccgcttcggcgg gctgggcctcatgggecttcetttcactgeccgetttccagtcgggaaacctgtcgtgccagctgcattaacatggtcatagctgtttcettgegtattggaccaggcg tttaagggcaccaataactgccttaaaaaaattacgeccegcctgccactcatcgcagtactgttgtaattcattaagcattctgccgacatggaagccatcacagac ggcatgatgaacctgaatcgccagcggcatcagcaccttgtcgecttgcgtataatattgcccatggtgaaaacgggggcgaagaagttgtccatattggccacgt ttaaatcaaaactggtgaaactcacccagggattggctgagacgaaaaacatattctcaataaaccetttagggaaataggccaggtttcaccgtaacacgccacat cttgcgaatatatgtgtagaaactgccggaaatcgtcgtggtattcactccagagcgatgaaaacgtttcagtttgctcatggaaaacggtgtaacaagggtgaacac tatcccatatcaccagctcaccgtctttcattgccatacggaattccggatgagcattcatcaggcgggcaagaatgtgaataaaggccggataaaacttgtgcttatt tttctttacggtctttaaaaaggccgtaatatccagctgaacggtctggttataggtacattgagcaactgactgaaatgcctcaaaatgttctttacgatgccattgggat atatcaacggtggtatatccagtgatttttttctccattttagcttccttagctcctgaaaatctcgataactcaaaaaatacgcccggtagtgatcttatttcattatggtgaa agttggaacctcttacgtgecgatcaacgtctcattttcgccaaaagttggeccagggettcceggtatcaacagggacaccaggatttatttattctgcgaagtgatct tccgagctcactcggtcgctacgcctccgggcgtgagactgcggcgggcgctagcggagtgtatactggcttactatgttggcactgatgagggtgtcagtgaagt gcttcatgtggcaggagaaaaaaggctgcaccggtgcgtcagcagaatatgtgatacaggatatattccgcttcctcgctcactgactcgctacgctcggtcgttcg actgcggcgagcggaaatggcttacgaacggggcggagatttcctggaagatgccaggaagatacttaacagggaagtgagagggccgcggcaaagccgtttt tccataggetccgecccctgacaagcatcacgaaatctgacgctcaaatcagtggtggcgaaaccegacaggactataaagataccaggcgtttcccctggcg gctccetcgtgcgctctcetgttcctgcctttcggtttaccggtgtcattccgctgttatggccgcgtttgtctcattccacgcctgacactcagttccgggtaggcagttc gctccaagctggactgtatgcacgaacccccgttcagtccgaccgetgcgecttatccggtaactatcgtcttgagtccaacceggaaagacatgcaaaagcacc actggcagcagccactggtaattgatttagaggagttagtcttgaagtcatgegccggttaaggctaaactgaaaggacaagtttggtgactgcgetcctccaagc cagttacctcggttcaaagagttggtagctcagagaaccttcgaaaaaccgccetgcaaggeggttttttcgttttcagagcaagagattacgcgcagaccaaaacg atctcaagaagatcatcttattaatcagataaaatattgctcatgagcccgaagtggcgagcccgatcttcccatcggtgatgtcggcgatataggcgccagcaac cgcacctgtggcgccggtgatgecggccacgatgcgtccggcgtagaggatctgctcatgtttgacagcttatcatcgatgcataatgtgcetgtcaaatggacga agcagggattctgcaaaccetatgctactccgtcaagccgtcaattgtctgattcgttaccaattatgacaacttgacggetacatcattcactttttcttcacaaccggc acggaactcgetcgggetggecceggtgcattttttaaataccegcgagaaatagagttgatcgtcaatcgcgatttgacaattaatcatccggctcgtataatgtgtg gaattgtgagcggataacaatttcacacaggagtaaaaatgggtagcagcgaagatcaggcatatcgtctgctgaatgattatgccaatggttttatggttagccagg ttctgtttgcagcatgtgaactgggtgtttttgatctgctggcagaagcaccgggtccgctggatgttgcagcagttgcagccggtgttcgtgcaagcgcacatggca ccgaactgctgctggatatttgtgttagcctgaaactgctgaaagttgaaacccgtggtggtaaagcattttatcgtaataccgaactgagcagcgattatctgaccac cgttagcccgaccagccagtgtagcatgctgaaatatatgggtcgtaccagctatcgttgttggggtcatctggcagatgcagttcgtgaaggtcgtaatcagtatct ggaaacctttggtgttccggcagaagaactgtttaccgcaatttatcgtagcgaaggtgaacgtctgcagtttatgcaggcactgcaagaggtttggagcgttaatgg tcgtagcgttctgaccgcatttgatctgagcgttttccgctgatgtgtgatctgggtggtggtgccggtgcactggcaaaagaatgtatgagcctgtatccgggttgta aaattaccgtttttgatattccggaagttgtgtggaccgcaaaacagcattttagctttcaagaagaggaacaaatcgactttcaagagggcgattttttcaaagatccg ctgccggaagcagatctgtatattctggcacgtgttctgcatgattgggcagatggtaaatgcagccatctgctggaacgtatttatcatacctgtaaaccgggtggtg gcattctggttattgaaagcetgctggatgaagatcgtcgtggtccgetgctgacccagctgtatagcctgaatatgctggttcagaccgaaggtcaagaacgtacc ccgacccattatcatatgctgctgagcagtgcaggttttcgtgattttcagttcaaaaaaaccggtgccatttatgatgcaatcctggcacgtaaataagtgccggatga tgcacatgacagat 


\section{$>$ pHM5}

ttccggtagtcaataaaccggtaaaccagcaatagacataagcggctatttaacgaccetgecetgaaccgacgaccgggtcatcgtggecggatcttgcggecc ctcggcttgaacgaattgttagacattatttgccgactaccttggtgatctcgcetttcacgtagtggacaaattcttccaactgatctgcgcgcgaggccaagcgatct tcttcttgtccaagataagcctgtctagcttcaagtatgacgggctgatactgggccggcaggcgctccattgcccagtcggcagcgacatccttcggcgcgattttg ccggttactgcgctgtaccaaatgcgggacaacgtaagcactacatttcgctcatcgccagccagtcgggcggcgagttccatagcgttaaggtttcatttagcgc ctcaaatagatcctgttcaggaaccggatcaaagagttcctccgccgetggacctaccaaggcaacgetatgttctcttgcttttgtcagcaagatagccagatcaat gtcgatcgtggctggctcgaagatacctgcaagaatgtcattgcgetgccattctccaaattgcagttcgcgettagctggataacgccacggaatgatgtcgtcgtg cacaacaatggtgacttctacagcgcggagaatctcgctctctccaggggaagccgaagtttccaaaaggtcgttgatcaaagctcgccgcgttgtttcatcaagcc ttacggtcaccgtaaccagcaaatcaatatcactgtgtggcttcaggccgccatccactgcggagccgtacaaatgtacggccagcaacgtcggttcgagatggc gctcgatgacgccaactacctctgatagttgagtcgatacttcggcgatcaccgcttccetcatactcttcctttttcaatattattgaagcatttatcagggttattgtctca tgagcggatacatatttgaatgtatttagaaaaataaacaaatagctagctcactcggtcgctacgctccgggcgtgagactgcggcgggcgctgcggacacatac aaagttacccacagattccgtggataagcaggggactaacatgtgaggcaaaacagcagggccgcgecggtggcgttttccataggctccgccetcctgccag agttcacataaacagacgcttttccggtgcatctgtgggagccgtgaggctcaaccatgaatctgacagtacgggcgaaaccegacaggacttaaagatccccac cgtttccggcgggtcgctccetcttgcgctctcetgttccgaccetgccgtttaccggatacctgttccgcetttctccettacgggaagtgtggcgctttctcatagctc acacactggtatctcggctcggtgtaggtcgttcgctccaagctgggctgtaagcaagaactcccegttcagcccgactgetgcgecttatccggtaactgttcactt gagtccaacccggaaaagcacggtaaaacgccactggcagcagccattggtaactgggagttcgcagaggatttgtttagctaaacacgcggttgctcttgaagt gtgcgccaaagtccggctacactggaaggacagatttggttgctgtgctctgcgaaagccagttaccacggttaagcagttcccaactgacttaaccttcgatcaa accacctcccaggtggtttttcgtttacagggcaaaagattacgcgcagaaaaaaggatctcaagaagatcctttgatcttttctactgaaccgctctagatttcag tgcaatttatctcttcaaatgtagcacctgaagtcagcccatacgatataagttgtaattctcatgttagtcatgcccegcgeccaccggaaggagctatcgcgagaa ttgaaggaaggccgtcaaggccgcatttgacaattaatcatccggctcgtataatgtgtggaattgtgagcggataacaatttcacacaggagtaaaaatgaaccgc atgaaaaacaactttcacatgctgcctgatgattttcgtgcagcaggtcataaagttattgattgggttgcagattatcatgcccacgttgaagattttcgcgttctgagc caggttaaaccgggtgaaatttgtgatggtctgccggatagccetccgcagcagggtgatagcgttaccaatattctgcctgatattgaacgtcatgttctgccaggta ttacccattggcagagcccgaacttttatgcatattttccgagcaataattccggtccgagcattctgggtgatctggttagcagcggtctgggtgttcagggtatgctg tgggcaaccagtccggcatgtaccgaagttgaaatgaaaatgctggattggctggttcagatgctgggcctgccggaacattttctgaatagcagcaaacatggtg gtggtgttattcaggatagcgcaagcagcgcaaccetgtgtgcactgctggcagcacgtgaacaggcaaccaatggtcagaccaatgaagaaggttgtcgtctgc cgctggtttgttataccagcaatcaggcacatagccatgttgagaaagatgttaaagttgcaggtctgggtcgtaaaaatctgcgtctgattgatgtggatcaagaattt gcaatgcgtccggaagcactggaacgtcagattgttgaagataaagcagcaggcaaaatcccgttttttgtttgtgcaaccattggtacaaccagcagcctggcaat tgatccgattccggaaattgcagccatttgtaaacgccatggtctgtggctgcatgttgatgcagcaatggcaggcaccgcagcactgtgtccggaatttcgttggac ccataatggtgttgaactggcagatagctatgcatttaatccgcacaaatggatgtataccaactttgattgtaccgccttttgggtgaaagatcgtcatgcactgattaa tagcctgagcgttgttccggaatatctgcgtaatcaggcaagcgaacagggtgaagtttttgattatcgtgattggcatgttccgctgggtcgtcgtttcgtgccetga aactgtggtttgtgattcgtcattatggtgtggaaggtctgcagcatcatgttcgtcagaatgttgcatgggcacaagaattcocagcatgggttaaagcagatagccg ttttgaactggttgcaccgcatccgctgagcctggtttgctttcgtctgaaaagcggtgatgcagccagcgaacagctgctgaaacgtgcaaatgaaagcggcaaa atctttatcagccataccaaactggatggcaaatatgttctgcgttttagcattggtcaggcaaaaaccgaacgtcatcatgttgaagccgcatggaaactgattagcg atctggccgatcgtagctaaaccgcagatgcactgaatggttaagtgccggatgatgcacttgacaattaatcatccggctcgtataatgtgtggaattgtgagcgga taacaatttcacacaggagtaaaaatgaacacctttcgtaccgcaaccgcacgtgatattccagatgttgcagcaaccetgaccgaagcatttgcgaccgatccgec taccagtgggtttttccggatggtacagcagccgttagccgttttttacccatgttgccgatcgtgttcataccgcaggeggtattgttgaactgctgccggatcgtg cagcaatgattgcactgectccgcatgttcgtctgccaggtgaagcagccgatggtcgtcaggcagaaattcagcgtcgtctggccgatcgccatccgctgactcc gcattattacetgctgtttatggtgtgcgtaccgcacatcagggtagtggectgggtggtcgtatgctggctcgtctgaccagccotgcagatcgtgatcgtgttggt acatataccgaagcaagcacctggegtggtgeccgtctgatgetgcgtcatggttttcatgccaccegtccgetgegectgecggatggtccgagcatgtttccget gtggcgtgatccgattcatgatcatagcgattaagtgccggatgatgcacctgggcctcatgggecttcetttcactgcccac 
$>$ pHM6

atctgtcatgtgcatcatccggcacttatttacgtgccaggattgcatcataaatggcaccggtttttttgaactgaaaatcacgaaaacetgcactgctcagcagcata tgataatgggtcggggtacgttcttgaccttcggtctgaaccagcatattcaggctatacagctgggtcagcagcggaccacgacgatcttcatccagcaggcttcca ataaccagaatgccaccacccggtttacaggtatgataaatacgttccagcagatggetgcatttaccatctgcccaatcatgcagaacacgtgccagaatatacag atctgcttccggcagcggatctttgaaaaaatcgccctcttgaaagtcgatttgttcctcttcttgaaagctaaaatgctgttttgcggtccacacaacttccggaatatca aaaacggtaattttacaacccggatacaggctcatacattcttttgccagtgcaccggcaccaccacccagatcacacatcagcggaaaaacgctcagatcaaatg cggtcagaacgctacgaccattaacgctccaaacctcttgcagtgcctgcataaactgcagacgttcacettcgctacgataaattgcggtaaacagttcttctgccg gaacaccaaaggtttccagatactgattacgaccttcacgaactgcatctgccagatgaccccaacaacgatagctggtacgacccatatatttcagcatgctacact ggctggtcgggctaacggtggtcagataatcgctgctcagttcggtattacgataaaatgctttaccaccacgggtttcaactttcagcagtttcaggctaacacaaat atccagcagcagttcggtgccatgtgcgcttgcacgaacaccggctgcaactgctgcaacatccagcggacccggtgettctgccagcagatcaaaaacaccca gttcacatgctgcaaacagaacctggctaaccataaaaccattggcataatcattcagcagacgatatgcetgatcttcgctgctacccattttactcctgtgtgaaatt gttatccgctcacaattccacacattatacgagccggatgattaattgtcaaatcgcgattgacgatcaactctattctcgcgggtatttaaaaaatgcaccggggcca gccegagcgagttccgtgccggttgtgaagaaaaagtgaatgatgtagccgtcaagttgtcataattggtaacgaatcagacaattgacggcttgacggagtagca tagggtttgcagaatccetgcttcgtccatttgacaggcacattatgcatcgatgataagctgtcaaacatgagcagatcctctacgccggacgcatcgtggccggc atcaccggcgecacaggtgcggttgetggcgectatatcgecgacatcaccgatggggaagatcgggetcgecacttcgggetcatgagcaaatattttatctgatt aataagatgatcttcttgagatcgttttggtctgcgcgtaatctcttgctctgaaaacgaaaaaaccgcttgcagggcggttttcgaaggttctctgagctaccaactc tttgaaccgaggtaactggcttggaggagcgcagtcaccaaaacttgtcctttcagtttagcettaaccggcgcatgacttcaagactaactcctctaaatcaattacc agtggctgctgccagtggtgcttttgcatgtcttccgggttggactcaagacgatagttaccggataaggcgcagcggtcggactgaacggggggttcgtgcata cagtccagcttggagcgaactgcctacceggaactgagtgtcaggcgtggaatgagacaaacgcggccataacagcggaatgacaccggtaaaccgaaaggc aggaacaggagagcgcacgagggagccgccagggggaaacgctggtatctttatagtcctgtcgggtttcgccaccactgatttgagcgtcagatttcgtgatg cttgtcaggggggcggagcctatggaaaaacggctttgccgcggcctctcacttcctgttaagtatcttcetggcatcttccaggaaatctccgccccgttcgtaa gccatttccgctcgccgcagtcgaacgaccgagcgtagcgagtcagtgagcgaggaagcggaatatatcctgtatcacatattctgctgacgcaccggtgcagcc ttttttctcctgccacatgaagcacttcactgacaccctcatcagtgccaacatagtaagccagtatacactccgctagcgeccgccgcagtctcacgcceggaggc gtagcgaccgagtgagctcggaagatcacttcgcagaataaataaatcctggtgtccetgttgataccgggaagccetgggecaacttttggcgaaaatgagacgt tgatcggcacgtaagaggttccaactttcaccataatgaaataagatcactaccgggcgtatttttgagttatcgagattttcaggagctaaggaagctaaaatggag aaaaaaatcactggatataccaccgttgatatatcccaatggcatcgtaaagaacattttgaggcatttcagtcagttgctcaatgtacctataaccagaccgttcagct ggatattacggcctttttaaagaccgtaaagaaaaataagcacaagttttatccggcctttattcacattcttgcccgectgatgaatgctcatccggaattccgtatggc aatgaaagacggtgagctggtgatatgggatagtgttcacccttgttacaccgttttccatgagcaaactgaaacgttttcatcgctctggagtgaataccacgacgat ttccggcagtttctacacatatattcgcaagatgtggcgtgttacggtgaaaacctggcctatttccctaaagggtttattgagaatatgtttttcgtctcagccaatccet gggtgagtttcaccagttttgatttaaacgtggccaatatggacaacttcttcgcccccgttttcaccatgggcaaatattatacgcaaggcgacaaggtgctgatgcc gctggcgattcaggttcatcatgccetctgtgatggcttccatgtcggcagaatgcttaatgaattacaacagtactgcgatgagtggcagggcggggcgtaatttttt taaggcagttattggtgcccttaaacgcctggtccaatacgcaaggaaacagctatgaccatgttaatgcagctggcacgacaggtttccegactggaaagcgggc agtgaaaggaaggccatgaggeccagccegccgaagcgggggtgccggtgatgtgaatcatccggcacttacggacgacgaaacagtgcttcggttttcgct gcacagatgaagtcattcagggtcagaccacctgcatcatgggtgctgaagttcacaactgcacgattataatgcacggacatatccggatgatgatcttcttgattg gcaatccatgccagtgcattaacaaatgccatggtttcatgatagttggcgaaacgataggttttacgcagttcaataccaacgatttcccaatccggcagggtgctca gcagctgtttcacggtattatctgccagcggactctgaccatgctgcggtgtacagctcagttccagcagattcatttttactcctgtcatgccgggtaataccggatag tcaatatgttctgttggacgtaacgataacacataaagtaaagttttcaatccatatttcgtacatttttatttacacaggcaatttagtcgcgctttcaaccettacctctgta tagataaatttacactcctatgcggcettgacggcettccttcaattcgccctatagtgagtcgtattacgtcgcgctcactggccgtcgttttacaacgtcgtgactgg gaaaacctggegttacccaacttaatcgecttgcagcacatcccetttcgecagctggegtaatagcgaagagggaccgtcggtcgcaatgttggttgacaatta atcatccggctcgtataatgtgtggaattgtgagcggataacaatttcacacaggagtaaaaatgaaactggaagatgttccgtggtttccgcgtaaaattagcgaact ggataaatgtagccatcgtgttctgatgtatggtagtgaactggatgcagatcatccgggttttaaagataatgtttatcgtcagcgtcgcaaatatttcgttgatgttgca atgggttacaaatatggtcagccgattccgcgtgttgaatataccgaagaagaaaccaaaacctggggtgttgttttcgtgaactgagcaaactgtatccgacccat gcatgtcgtgaatatctgaaaaactttccgetgetgaccaaatattgtggttatcgtgaagataacgttccgcagctggaagatgtgagcatgtttctgaaagaacgta gcggttttaccgttcgtccggttgcaggttatctgagtccgcgtgatttctggcaggtctggcatatcgtgtttttcattgtacccagtatattcgccatggtagcgatcc gctgtatactccggaaccggatacctgtcatgaactgctgggtcatgtgccgctgctggcagatccgaaatttgcacagtttagccaagaaattggtctggcaagcet gggtgcaagtgatgaagatgtgcagaaactggcaacctgttatttctttaccattgaattggcctgtgcaaacaagagggtcagctgcgtgcatatggtgcaggtct gctgagcagcattggtgaactgaaacatgcactgagcgataaagcatgtgttaaagcatttgatccgaaaaccacctgtctgcaagaatgtctgattaccaccttca agaagcctatttcgttagcgaaagctttgaagaggccaaagaaaaatgcgcgattttgccaaaagcattacccgtccgtttagcgtttatttcaatccgtatacccag agcatcgagatcctgaaagatacctaagtgccggatgatgcacttacgcgcagcgtgaccgctacacttgccagcgectagcgecegctcetttcgctttcttcet agggataacaggg 


\section{$>$ pHM18}

atctgtcatgtgcatcatccggcacttatttacgtgccaggattgcatcataaatggcaccggttttttgaactgaaaatcacgaaaacctgcactgctcagcagcata tgataatgggtcggggtacgttcttgaccttcggtctgaaccagcatattcaggctatacagctgggtcagcagcggaccacgacgatcttcatccagcaggctttca ataaccagaatgccaccacceggtttacaggtatgataaatacgttccagcagatggctgcatttaccatctgcccaatcatgcagaacacgtgccagaatatacag atctgcttccggcagcggatctttgaaaaaatcgecctcttgaaagtcgatttgttcctcttcttgaaagctaaaatgctgttttgcggtccacacaacttccggaatatca aaaacggtaattttacaacccggatacaggctcatacattcttttgccagtgcaccggcaccaccacccagatcacacatcagcggaaaaacgctcagatcaaatg cggtcagaacgctacgaccattaacgctccaaacctcttgcagtgcctgcataaactgcagacgttcaccttcgctacgataaattgcggtaaacagttcttctgccg gaacaccaaaggtttccagatactgattacgaccttcacgaactgcatctgccagatgaccccaacaacgatagctggtacgacccatatatttcagcatgctacact ggctggtcgggctaacggtggtcagataatcgctgctcagttcggtattacgataaaatgctttaccaccacgggtttcaactttcagcagtttcaggctaacacaaat atccagcagcagttcggtgccatgtgcgcttgcacgaacaccggctgcaactgctgcaacatccagcggacceggtgcttctgccagcagatcaaaaacaccca gttcacatgctgcaaacagaacctggctaaccataaaaccattggcataatcattcagcagacgatatgcctgatcttcgctgctacccattttactcetgtgtgaaatt gttatccgctcacaattccacacattatacgagccggatgattaattgtcaaatcgegattgacgatcaactctatttctcgcgggtatttaaaaaatgcaccggggcca gcccgagcgagttccgtgccggttgtgaagaaaaagtgaatgatgtagccgtcaagttgtcataattggtaacgaatcagacaattgacggcttgacggagtagca tagggtttgcagaatccetgcttcgtccatttgacaggcacattatgcatcgatgataagctgtcaaacatgagcagatcctctacgecggacgcatcgtggceggc atcaccggcgccacaggtgcggttgctggegectatatcgccgacatcaccgatggggaagatcgggctcgccacttcgggctcatgagcaaatatttatctgatt aataagatgatcttcttgagatcgttttggtctgcgcgtaatctcttgctctgaaaacgaaaaaaccgccttgcagggeggttttcgaaggttctctgagctaccaactc tttgaaccgaggtaactggettggaggagcgcagtcaccaaaacttgtcctttcagtttagcettaaccggcgcatgacttcaagactaactcctctaaatcaattacc agtggctgctgccagtggtgctttgcatgtcttccgggttggactcaagacgatagttaccggataaggcgcagcggtcggactgaacggggggttcgtgcata cagtccagcttggagcgaactgcctacceggaactgagtgtcaggcgtggaatgagacaaacgcggccataacagcggaatgacaccggtaaaccgaaaggc aggaacaggagagcgcacgagggagccgccagggggaaacgcctggtatctttatagtcctgtcgggtttcgccaccactgatttgagcgtcagatttcgtgatg cttgtcaggggggcggagcctatggaaaaacggctttgccgcggccetctcacttccetgttaagtatcttcetggcatcttccaggaaatctccgcccegttcgtaa gccatttccgctcgccgcagtcgaacgaccgagcgtagcgagtcagtgagcgaggaagcggaatatatcctgtatcacatattctgctgacgcaccggtgcagcc tttttctcctgccacatgaagcacttcactgacaccctcatcagtgccaacatagtaagccagtatacactccgctagcgccegcegcagtctcacgeccggaggc gtagcgaccgagtgagctcggaagatcacttcgcagaataaataaatcctggtgtccetgttgataccgggaagccctgggccaacttttggcgaaaatgagacgt tgatcggcacgtaagaggttccaactttcaccataatgaaataagatcactaccgggcgtatttttgagttatcgagatttcaggagctaaggaagctaaaatggag aaaaaatcactggatataccaccgttgatatatcccaatggcatcgtaaagaacattttgaggcatttcagtcagttgctcaatgtacctataaccagaccgttcagct ggatattacggectttttaaagaccgtaaagaaaaataagcacaagttttatccggectttattcacattcttgccegcctgatgaatgctcatccggaattccgtatggc aatgaaagacggtgagctggtgatatgggatagtgttcaccettgttacaccgttttccatgagcaaactgaaacgtttcatcgctctggagtgaataccacgacgat ttccggcagtttctacacatatattcgcaagatgtggegtgttacggtgaaaacctggectatttccctaaagggtttattgagaatatgttttcgtctcagccaatccct gggtgagtttcaccagttttgatttaaacgtggccaatatggacaacttcttcgecccegttttcaccatgggcaaatattatacgcaaggcgacaaggtgctgatgcc gctggcgattcaggttcatcatgccgtctgtgatggcttccatgtcggcagaatgcttaatgaattacaacagtactgcgatgagtggcagggeggggegtaatttttt taaggcagttattggtgccettaaacgcctggtccaatacgcaaggaaacagctatgaccatgttaatgcagctggcacgacaggtttccegactggaaagcgggc agtgaaaggaaggcccatgaggcccagcccgccgaagcgggggtgccggtgatgtgaatcatccggcacttacggacgacgaaacagtgcttcggtttcgct gcacagatgaagtcattcagggtcagaccacctgcatcatgggtgctgaagttcacaactgcacgattataatgcacggacatatccggatgatgatcttcttgattg gcaatccatgccagtgcattaacaaatgccatggtttcatgatagttggcgaaacgataggttttacgcagttcaataccaacgatttcccaatccggcagggtgctca gcagctgtttcacggtattatctgccagcggactctgaccatgctgcggtgtacagctcagttccagcagattcattttactcetgtcatgecgggtaataccggatag tcaatatgttctgttggacgtaacgataacacataaagtaaagtttcaatccatatttcgtacatttttatttacacaggcaatttagtcgcgetttcaaccettacctctgta tagataaatttacactccetatgcggcettgacggccttccttcaattcgccctatagtgagtcgtattacgtcgcgctcactggccgtcgttttacaacgtcgtgactgg gaaaecctggegttacccaacttaatcgecttgcagcacatcccectttcgccagctggcgtaatagcgaagagggaccgtcggtcgcaatgttggttgacaatta atcatccggctcgtataatgtgtggaattgtgagcggataacaatttcacacaggagtaaaaatgaaactggaagatgttccgtggtttccgcgtaaaattagcgaact ggataaatgtagccatcgtgttctgatgtatggtagtgaactggatgcagatcatccgggttttaaagataatgtttatcgtcagcgtcgcaaatatttcgttgatgttgca atgggttacaaatatggtcagccgattccgcgtgttgaatataccgaagaagaaaccaaaacctggggtgttgttttcgtgaactgagcaaactgtatccgacccat gcatgtcgtgaatatctgaaaatctttgcctgctgaccaaatattgtggttatcgtgaagataacgttccgcagctggaagatgtgagcatgtttctgaaagaacgtag cggttttaccgttcgtccggttgcaggttatctgagtccgcgtgattttctggcaggtctggcatatcgtgttttcattgtacccagtatattcgccatggtagcgatccg ctgtatactccggaaccggatacctgtcatgaactgctgggtcatgtgccgctgctggcagatccgaaatttgcacagtttagccaagaaattggtctggcaagcctg ggtgcaagtgatgaagatgtgcagaaactggcaacctgttatttctttaccattgaatttggcetgtgcaaacaagagggtcagctgcgtgcatatggtgcaggtctg ctgagcagcattggtgaactgaaacatgcactgagcgataaagcatgtgttaaagcatttgatccgaaaaccacctgtctgcaagaatgtctgattaccacctttcaa gaagcctatttcgttagcgaaagctttgaagaggccaaagaaaaaatgcgcgattttgccaaaagcattacccgtccgtttagcgtttatttcaatccgtatacccaga gcatcgagatcetgaaagatacctaagtgccggatgatgcacttacgcgcagcgtgaccgctacacttgccagcgecctagcgecegctcetttegctttcttccta gggataacaggg 
>pHM39

ttccggtagtcaataaaccggtaaaccagcaatagacataagcggctatttaacgaccetgccetgaaccgacgaccgggtcatcgtggceggatcttgcggecc ctcggcttgaacgaattgttagacattatttgccgactaccttggtgatctcgectttcacgtagtggacaaattcttccaactgatctgcgegcgaggccaagcgatct tcttcttgtccaagataagcetgtctagcttcaagtatgacgggetgatactgggccggcaggcgctccattgcccagtcggcagcgacatccttcggegcgattttg ccggttactgcgctgtaccaaatgcgggacaacgtaagcactacatttcgctcatcgccagcccagtcgggeggcgagttccatagcgttaaggtttcatttagcgc ctcaaatagatcctgttcaggaaccggatcaaagagttcctccgccgctggacctaccaaggcaacgctatgttctcttgctttgtcagcaagatagccagatcaat gtcgatcgtggctggctcgaagatacctgcaagaatgtcattgcgctgccattctccaaattgcagttcgcgcttagctggataacgccacggaatgatgtcgtcgtg cacaacaatggtgacttctacagcgcggagaatctcgctctctccaggggaagccgaagtttccaaaaggtcgttgatcaaagctcgcegegttgtttcatcaagcc ttacggtcaccgtaaccagcaaatcaatatcactgtgtggcttcaggccgccatccactgcggagccgtacaaatgtacggccagcaacgtcggttcgagatggc gctcgatgacgccaactacctctgatagttgagtcgatacttcggcgatcaccgcttccetcatactcttccttttcaatattattgaagcatttatcagggttattgtctca tgagcggatacatatttgaatgtatttagaaaaataaacaaatagctagctcactcggtcgctacgctccgggcgtgagactgcggcgggcgetgcggacacatac aaagttacccacagattccgtggataagcaggggactaacatgtgaggcaaaacagcagggccgcgccggtggcgttttccataggctccgccctcctgccag agttcacataaacagacgcttttceggtgcatctgtgggagccgtgaggctcaaccatgaatctgacagtacgggcgaaacccgacaggacttaaagatccccac cgtttccggcgggtcgctccetcttgegctctcctgttccgaccetgccgtttaccggatacctgttcegcetttctccettacgggaagtgtggegetttctcatagctc acacactggtatctcggctcggtgtaggtcgttcgctccaagctgggctgtaagcaagaactccccgttcagcccgactgctgcgccttatccggtaactgttcactt gagtccaacccggaaaagcacggtaaaacgccactggcagcagccattggtaactgggagttcgcagaggatttgtttagctaaacacgcggttgctcttgaagt gtgcgccaaagtccggctacactggaaggacagatttggttgctgtgctctgcgaaagccagttaccacggttaagcagttccccaactgacttaaccttcgatcaa accacctccccaggtggtttttcgtttacagggcaaaagattacgcgcagaaaaaaaggatctcaagaagatcctttgatctttctactgaaccgctctagatttcag tgcaatttatctcttcaaatgtagcacctgaagtcagccccatacgatataagttgtaattctcatgttagtcatgcccegcgcccaccggaaggagctatcgcgagaa ttgaaggaaggccgtcaaggccgcattaccgcagatgcactgaatggttaagtgccggatgatgcacttgacaattaatcatccggctcgtataatgtgtggaattgt gagcggataacaatttcacacaggagtaaaaatgaacacctttcgtaccgcaaccgcacgtgatattccagatgttgcagcaaccctgaccgaagcatttgcgacc gatccgcctacccagtgggtttttceggatggtacagcagccgttagccgttttttacccatgttgccgatcgtgttcataccgcaggcggtattgttgaactgetgec ggatcgtgcagcaatgattgcactgcctccgcatgttcgtctgccaggtgaagcagccgatggtcgtcaggcagaaattcagcgtcgtctggecgatcgccatccg ctgactccgcattattacetgctgtttatggtgtgcgtaccgcacatcagggtagtggectgggtggtcgtatgctggctcgtctgaccagccgtgcagatcgtgatc gtgttggtacatataccgaagcaagcacctggcgtggtgccegtctgatgctgcgtcatggttttcatgccaccegtccgctgegcetgecggatggtccgagcatg tttccgctgtggcgtgatccgattcatgatcatagcgattaagtgccggatgatgcacctgggectcatgggecttcctttcactgcccac 


\section{>pHM64}

atctgtcatgtgcatcatccggcacttatttacgtgccaggattgcatcataaatggcaccggttttttgaactgaaaatcacgaaaacctgcactgctcagcagcata tgataatgggtcggggtacgttcttgaccttcggtctgaaccagcatattcaggctatacagctgggtcagcagcggaccacgacgatcttcatccagcaggctttca ataaccagaatgccaccacceggtttacaggtatgataaatacgttccagcagatggctgcatttaccatcttcccaatcatgcagaacacgtgccagaatatacaga tctgcttccggcagcggatcttgaaaaaatcgecctcttgaaagtcgatttgttcctcttcttgaaagctaaaatgctgttttgcggtccacacaacttccggaatatcaa aaacggtaattttacaacccggatacaggctcatacattcttttgccagtgcaccggcaccaccacccagatcacacatcagcggaaaaacgctcagatcaaatgc ggtcagaacgctacgaccattaacgctccaaacctcttgcagtgcctgcataaactgcagacgttcaccttcgctacgataaattgcggtaaacagttcttctgccgg aacaccaaaggtttccagatactgattacgaccttcacgaactgcatctgccagatgaccccaacaacgatagctggtacgacccatatattcagcatgctacactg gctggtcgggctaacggtggtcagataatcgctgctcagttcggtattacgataaaatgctttaccaccacgggtttcaactttcagcagtttcaggctaacacaaatat ccagcagcagttcggtgccatgtgcgcttgcacgaacaccggctgcaactgctgcaacatccagcggacceggtgcttctgccagcagatcaaaaacacccagtt cacatgctgcaaacagaacctggctaaccataaaaccattggcataatcattcagcagacgatatgcetgatcttcgctgctacccattttactcetgtgtgaaattgtt atccgctcacaattccacacattatacgagccggatgattaattgtcaaatcgcgattgacgatcaactctattctcgcgggtatttaaaaaatgcaccggggecagc ccgagcgagttccgtgccggttgtgaagaaaaagtgaatgatgtagccgtcaagttgtcataattggtaacgaatcagacaattgacggcttgacggagtagcata gggtttgcagaatccetgcttcgtccatttgacaggcacattatgcatcgatgataagctgtcaaacatgagcagatcctctacgccggacgcatcgtggccggcat caccggcgccacaggtgcggttgctggcgectatatcgccgacatcaccgatggggaagatcgggctcgccacttcgggctcatgagcaaatatttatctgatta ataagatgatcttcttgagatcgttttggtctgcgcgtaatctcttgctctgaaaacgaaaaaaccgcttgcagggcggttttcgaaggttctctgagctaccaactct ttgaaccgaggtaactggcttggaggagcgcagtcaccaaaacttgtcctttcagtttagccttaaccggcgcatgacttcaagactaactcctctaaatcaattacca gtggctgctgccagtggtgcttttgcatgtctttcegggttggactcaagacgatagttaccggataaggcgcagcggtcggactgaacggggggttcgtgcatac agtccagcttggagcgaactgcctacceggaactgagtgtcaggegtggaatgagacaaacgeggccataacagcggaatgacaccggtaaaccgaaaggca ggaacaggagagcgcacgagggagccgccagggggaaacgcctggtatctttatagtcctgtcgggtttcgccaccactgatttgagcgtcagatttcgtgatgct tgtcaggggggcggagcctatggaaaaacggctttgccgcggecctctcacttccetgttaagtatcttcctggcatcttccaggaaatctccgcccegttcgtaagc catttcegctcgccgcagtcgaacgaccgagcgtagcgagtcagtgagcgaggaagcggaatatatcctgtatcacatattctgctgacgcaccggtgcagccttt tttctcctgccacatgaagcacttcactgacaccetcatcagtgccaacatagtaagccagtatacactccgctagcgcccgccgcagtctcacgcccggaggcgt agcgaccgagtgagctcggaagatcacttcgcagaataaataaatcctggtgtccetgttgataccgggaagccetgggccaacttttggcgaaaatgagacgttg atcggcacgtaagaggttccaactttcaccataatgaaataagatcactaccgggcgtatttttgagttatcgagattttcaggagctaaggaagctaaaatggagaa aaaaatcactggatataccaccgttgatatatcccaatggcatcgtaaagaacattttgaggcatttcagtcagttgctcaatgtacctataaccagaccgttcagctgg atattacggcctttttaaagaccgtaaagaaaaataagcacaagttttatccggcctttattcacattcttgccegcctgatgaatgctcatccggaattccgtatggcaa tgaaagacggtgagctggtgatatgggatagtgttcaccettgttacaccgtttccatgagcaaactgaaacgtttcatcgctctggagtgaataccacgacgatttc cggcagtttctacacatatattcgcaagatgtggegtgttacggtgaaaacctggcctatttccctaaagggtttattgagaatatgttttcgtctcagccaatccetgg gtgagtttcaccagttttgatttaaacgtggccaatatggacaacttcttcgcccccgtttcaccatgggcaaatattatacgcaaggcgacaaggtgctgatgccgct ggcgattcaggttcatcatgccgtctgtgatggcttccatgtcggcagaatgcttaatgaattacaacagtactgegatgagtggcagggcggggcgtaattttttaa ggcagttattggtgccettaaacgcctggtccaatacgcaaggaaacagctatgaccatgttaatgcagctggcacgacaggtttcccgactggaaagcgggcagt gaaaggaaggcccatgaggcccagcccgccgaagcgggggtgccggtgatgtgaatcatccggcacttacggacgacgaaacagtgcttcggtttcgctgca cagatgaagtcattcagggtcagaccacctgcatcatgggtgctgaagttcacaactgcacgattataatgcacggacatatccggatgatgatcttcttgattggca atccatgccagtgcattaacaaatgccatggtttcatgatagttggcgaaacgataggttttacgcagttcaataccaacgatttcccaatccggcagggtgctcagca gctgtttcacggtattatctgccagcggactctgaccatgetgeggtgtacagctcagttccagcagattcattttactcctgtcatgccgggtaataccggatagtca atatgttctgttggacgtaacgataacacataaagtaaagtttcaatccatatttcgtacattttatttacacaggcaatttagtcgcgetttcaaccettacctctgtatag ataaatttacactccetatgcggcettgacggecttccttcaattcgccctatagtgagtcgtattacgtcgegctcactggccgtcgttttacaacgtcgtgactggga aaaccetggegttacccaacttaatcgecttgcagcacatccccetttcgecagctggegtaatagcgaagagggaccgtcggtcgcaatgttggttgacaattaat catccggctcgtataatgtgtggaattgtgagcggataacaatttcacacaggagtaaaaatgaaactggaagatgttccgtggtttccgcgtaaaattagcgaactg gataaatgtagccatcgtgttctgatgtatggtagtgaactggatgcagatcatccgggttttaaagataatgtttatcgtcagcgtcgcaaatatttcgttgatgttgcaa tgggttacaaatatggtcagccgattccgegtgttgaatataccgaagaagaaaccaaaacctggggtgttgttttcgtgaactgagcaaactgtatccgacccatg catgtcgtgaatatctgaaaatctttgcctgctgaccaaatattgtggttatcgtgaagataacgttccgcagctggaagatgtgagcatgtttctgaaagaacgtagc ggttttaccgttcgtccggttgcaggttatctgagtccgcgtgatttctggcaggtctggcatatcgtgttttcattgtacccagtatattcgccatggtagcgatccgct gtatactccggaaccggatacctgtcatgaactgctgggtcatgtgccgctgctggcagatccgaaatttgcacagtttagccaagaaattggtctggcaagcetgg gtgcaagtgatgaagatgtgcagaaactggcaacctgttatttctttaccattgaatttggcctgtgcaaacaagagggtcagctgcgtgcatatggtgcaggtctgct gagcagcattggtgaactgaaacatgcactgagcgataaagcatgtgttaaagcatttgatccgaaaaccacctgtctgcaagaatgtctgattaccacctttcaaga agcctatttcgttagcgaaagctttgaagaggccaaagaaaaaatgcgcgattttgccaaaagcattaccegtccgtttagcgtttatttcaatccgtatacccagagc atcgagatcctgaaagatacctaagtgccggatgatgcacttacgegcagcgtgaccgctacacttgccagcgecctagcgccegctcetttcgctttcttcetagg gataacaggg 